\title{
Quantitative Mapping of Waste Coal Tar deposits by EM Conductivity and VLF-Resistivity Surveys
}

\author{
R D Ogilvy ${ }^{1}$, P I Meldrum ${ }^{1}$ and J Nissen ${ }^{2}$ \\ 1 - British Geological Survey, Keyworth, Nottingham NG12 5GG, UK \\ 2 - ABEM Instrument AB, P O Box 20086, S -161 02 Bromma, Sweden
}

The nature of coal tar deposits presents considerable difficulties for traditional site investigation methods such as boreholes and cone penetrometer tests. These intrusive methods normally require access by heavy vehicles and would be at risk on a tar surface. However, the low viscosity of $\operatorname{tar}$ (in winter) means site characterisation may be undertaken by a geophysicist using lightweight instrumentation.

A geophysical survey is described which shows how the extent and depth of waste tar pits were determined by EM31/34 conductivity and VLF-Resistivity surveys . 1D and 2D computational modelling techniques were used to determine the approximate $3 \mathrm{D}$ spatial distribution of the tar and hence the quantity of tar present. This information was required to assist decision making on the most appropriate remediation strategy. The tar was acidic in nature and relatively conductive (5 - 12 ohm.m) compared to the host mudstone (50 -100 ohm.m). Numerical modelling indicated that a highly conductive tar leachate may be present at the base of the tarpits. Support for this model was provided by laboratory tests which showed that leachate from the tar could have an electrical conductivity of $\sim 300 \mathrm{mS} / \mathrm{m}(3.3$ ohm.m). Iron-rich furnace slag was extensively used as back-fill to cover the tarpits and this was expected to significantly enhance the free ion concentration of any aqueous fluid. The quantitative modelling indicated an in-situ leachate resistivity as low as $0.5-1 \mathrm{ohm} . \mathrm{m}$.

The results confirmed that EM31/34 conductivity soundings were a cost-effective and reliable method for mapping the depth and lateral extent of the tarpits. An approximate 2D geoelectric section was built up from the independent 1D sounding solutions obtained at each station. This approach was valid where the earth was essentially stratiform within the sampling volume of the dipole system (eg: over the centre of the tarpits) but clearly had restricted reliability over the tarpit edges. Shallow thicknesses of overlying backfill $(<2 \mathrm{~m})$ could not be resolved by these instruments due to their low excitation frequencies and large dipole spacings but depth scans down to $25 \mathrm{~m}$ were obtained. VLF-Resistivity trials showed that this method, although prone to cultural noise, may have distinct advantages for such problems. VLF-R is operationally more rapid than EM34 sounding and the results can be modelled in terms of $2 \mathrm{D}$ geology. The phase angle parameter was found to be particularly sensitive to the presence of thin leachate plumes (see Fig. 1). Removal of the leachate horizon resulted in a phase reversal and a serious model misfit. Conversely, the apparent resistivity response was primarily influenced by the bulk tar resistivities of $5-12 \mathrm{ohm} . \mathrm{m}$. Based on the quantitative models obtained, the volume of tar in each pit could be calculated. 
The total volume of tar on the site was estimated to be of the order of $50,324 \mathrm{~m}^{3}$. The results highlight the importance of multi-component analysis and the advantages of non-invasive geophysical methods for waste site characterisation.

Fig. 1

2D VLF-R INTERPRETATION ( FILES = cinvlfp.dat, cinvlfr.dat )

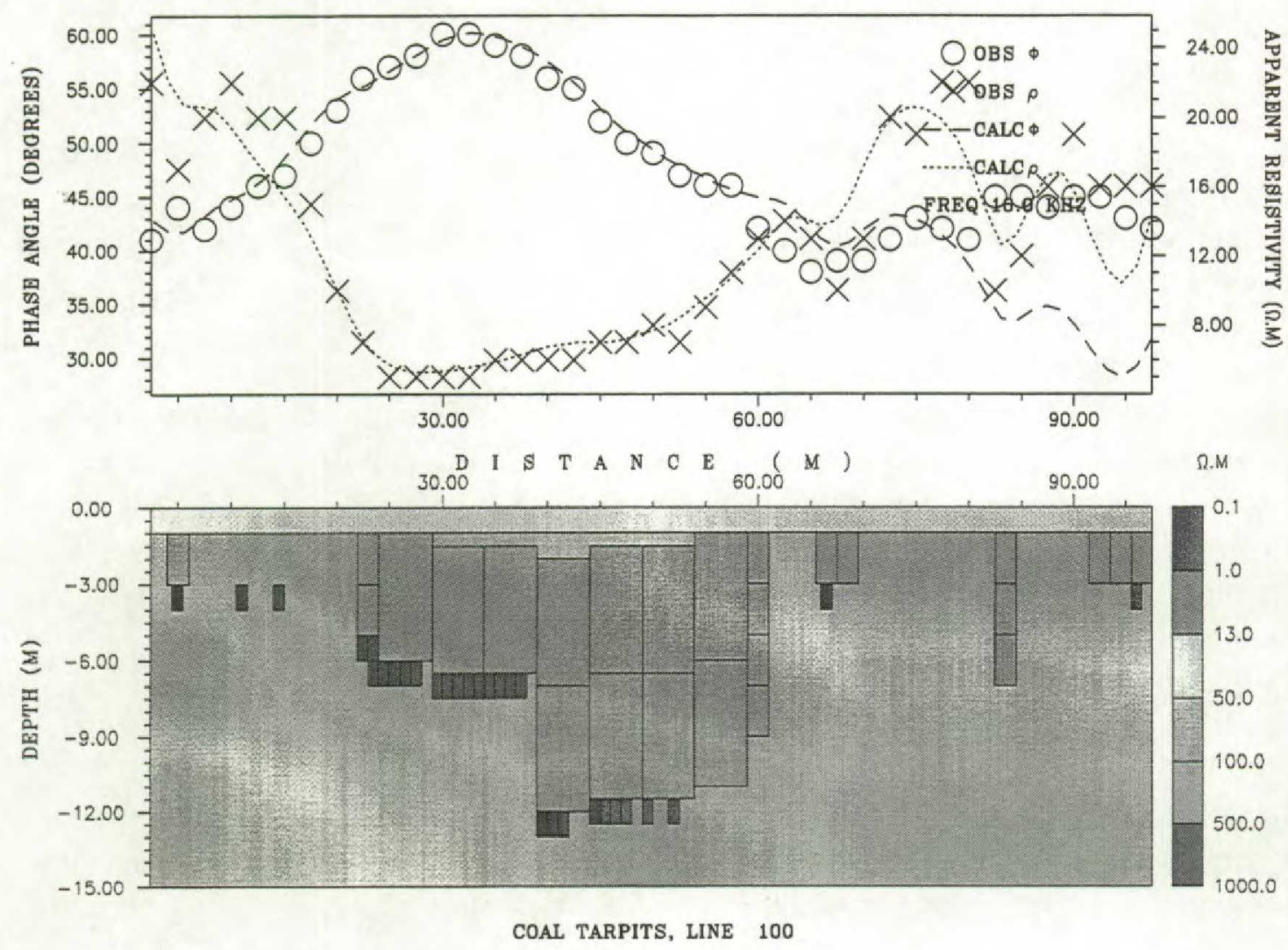

